\title{
Extraction of Some New Solutions of Linear Differential Equations by Dual Space
}

\author{
Gülay KorU YüceKaya (DD \\ Selcuk University, Faculty of Science, Department of Mathematics, Alaeddin Keykubat Campus, 42130, Konya-Turkey
}

Received: 09-10-2020 • Accepted: 14-12-2020

\begin{abstract}
AвSTRACT. In this study, the solutions of second order linear homogeneous ordinary differential equations being variable and constant coefficients have been obtained by using power series method which is used frequently in dual space. The solutions of second order homogeneous ordinary differential equations being constant and variable coefficients in dual space by using power series have given and some applications have implemented.
\end{abstract}

2010 AMS Classification: 51J15, 53A17

Keywords: Dual number, Dual function, Analytic function, Linear differential equation, Power series.

\section{INTRODUCTION}

In dual space, many works have been carried on dual numbers, dual vectors, dual functions and their applications. In [1], any differential equation is considered; the differential equation in the dual part is divided into real and dual parts such that each term of the differential equation in the real part has an upper order differential. When the equations in these parts are solved separately, it is seen that the solution of the dual part is found by adding a constant to the solution of the real part. Thus, by using this method, it has been seen that the solution of a higher order differential equation is easily obtained by reducing the orders of each term of the differential equation. Information about the dual numbers, dual space and functions with dual variables used in this study have been presented in [3,4]. By introducing second order linear differential equations with variable and constant coefficients in dual space in [6], their solutions have been investigated by using power series. In this study, second order homogeneous ordinary differential equations with variable and constant coefficients in dual space and their solutions have been studied by using power series in detail, and also simulations have been given.

\section{Preliminary}

In this part, it will be given some descriptions and theorem which will be in this article.

Definition 2.1 ( $[9,10])$. Each element of $\mathbb{D}=\left\{Z=\left(x, x^{*}\right): Z=x+\varepsilon x^{*}, \varepsilon \neq 0, \varepsilon^{2}=0, x, x^{*} \in \mathbb{R}\right\}$ where $\mathbb{D}=\mathbb{R} \times \mathbb{R}$ is called dual number. Here, $x \in \mathbb{R}$ is the real part of $Z ; x^{*} \in \mathbb{R}$ is the imajinary part of $Z$ and it is shown by $\operatorname{Re}(Z)=x, D u(Z)=x^{*}$.

Email address: gulay.koru@selcuk.edu.tr (G. Koru Yücekaya) 
Theorem 2.2 ( [4]). For $Z_{1}=\left(x+\varepsilon x^{*}\right) \in \mathbb{D}, Z_{2}=\left(x+\varepsilon x^{*}\right) \in \mathbb{D}$ addition and multiplication is defined by

$$
\begin{aligned}
Z_{1}+Z_{2} & =\left(x_{1}+x_{2}\right)+\varepsilon\left(x_{1}^{*}+x_{2}^{*}\right), \\
Z_{1} \cdot Z_{2} & =x_{1} x_{2}+\varepsilon\left(x_{1} x_{2}^{*}+x_{2} x_{1}^{*}\right) .
\end{aligned}
$$

In addition to this, $(\mathbb{D},+,$.$) triple is a ring but not a field.$

Theorem 2.3 ( [4]). Dual number ring $(\mathbb{D},+,$.$) includes a subset as a subfield which is isomorphic \mathbb{R}$ to real number set.

Definition 2.4 ( $[9,10])$. Let $\mathbb{D}_{1}$ and $\mathbb{D}_{2}$ are dual points sets. If for $\forall Z \in \mathbb{D}_{1}$ point is in reply to $W \in \mathbb{D}_{2}$ dual number with any $f$ rule where $Z=\left(x+\varepsilon x^{*}\right) \in \mathbb{D}_{1}$ an arbitrary dual variety, $f$ is called dual function which is from $\mathbb{D}_{1}$ to $\mathbb{D}_{2}$. It is shown by

$$
f: \mathbb{D}_{1} \rightarrow \mathbb{D}_{2}, f(Z)=W .
$$

$\mathbb{D}_{1}$ is the definition of $f$ and $\mathbb{D}_{2}$ is value region defion over $\mathbb{D}_{1}$. If $Z=x+\varepsilon x^{*}$ is written in $W=f(Z)$ and $\operatorname{Re}(W)=u\left(x, x^{*}\right), D u(W)=u^{*}\left(x, x^{*}\right)$.

$$
f(Z)=u\left(x, x^{*}\right)+\varepsilon u^{*}\left(x, x^{*}\right)
$$

is found. Than $f=u+\epsilon u^{*}$ is written where $u: \mathbb{R} \times \mathbb{R} \rightarrow \mathbb{R}$ and $u^{*}: \mathbb{R} \times \mathbb{R} \rightarrow \mathbb{R}$ real two variables function. If $f$ is analytic,

$$
u\left(x, x^{*}\right)=f(x) \quad \text { and } \quad u^{*}\left(x, x^{*}\right)=x^{*} f(x) .
$$

Definition 2.5 ( [4]). Let $f$ dual function defines in a $G$ district. If it is found $\delta>0$ for $\forall \eta>0$, for all $0<|h|<\delta$ such that

$$
\left|\frac{f\left(Z_{0}+h\right)-f\left(Z_{0}\right)}{h}-f^{\prime}\left(Z_{0}\right)\right|<\eta
$$

exists $f^{\prime}\left(Z_{0}\right)$, then it is called differentiable at $Z_{0} \in G$ point. The derive of $f$ at $Z_{0}$ is

$$
f^{\prime}\left(Z_{0}\right)=\lim _{h \rightarrow 0} \frac{f\left(Z_{0}+h\right)-f\left(Z_{0}\right)}{h} .
$$

The derivative rules of the addition, multiplication and divison of dual functions $f=u+\epsilon u^{*}$ and $g=v+\varepsilon v^{*}$ are the same as real functions.

Definition 2.6 ( [4]). If dual function $f$ can be differentiable at every point of $G$ district, than $G$ is analytic and regular.

Definition 2.7 ( [4]). Suppose that an infinite $f_{0,} f_{1}, f_{2}, \ldots, f_{n}, \ldots$ which is occured by arbitrary dual functions. Let $Z=x+\varepsilon x^{*}$ is a point which belongs to definition district of all these functions. At this point,

$$
f_{0}(Z)+f_{1}(Z)+f_{2}(Z)+\cdots+f_{n}(Z)+\cdots=\sum_{n=0}^{\infty} f_{n}(Z)
$$

series is convergent or not. The point set of this convergent series a called convergence district of series.

Theorem 2.8 ( [4]). If $n \in N$ than

$$
Z^{n}=\left(x+\varepsilon x^{*}\right)^{n}=x^{n}+\varepsilon n x^{n-1} x^{*}
$$

Definition 2.9 ( [4]).

$$
f(Z)=f\left(Z_{0}\right)+\frac{Z-Z_{0}}{1 !} f^{\prime}\left(Z_{0}\right)+\frac{\left(Z-Z_{0}\right)^{2}}{2 !} f^{\prime \prime}\left(Z_{0}\right)+\cdots+\frac{\left(Z-Z_{0}\right)^{n}}{n !} f^{(n)}\left(Z_{0}\right)+\cdots
$$

series is called the Taylor expansion of $f$ dual function at $Z_{0} \in G$ is

or

$$
f\left(x+\varepsilon x^{*}\right)=f(0)+\frac{x+\varepsilon x^{*}}{1 !} f^{\prime}(0)+\frac{\left(x+\varepsilon x^{*}\right)^{2}}{2 !} f^{\prime \prime}(0)+\cdots+\frac{\left(x+\varepsilon x^{*}\right)^{n}}{n !} f^{(n)}(0)+\cdots
$$

$$
\begin{aligned}
f\left(x+\varepsilon x^{*}\right)= & f(0)+\frac{x}{1 !} f^{\prime}(0)+\frac{x^{2}}{2 !} f^{\prime \prime}(0)+\cdots+\frac{x^{n-1}}{(n-1) !} f^{(n-1)}(0)+\frac{x^{n}}{n !} f^{(n)}(0)+ \\
& \cdots+\varepsilon x^{*}\left[f^{\prime}(0)+\frac{x}{1 !} f^{\prime \prime}(0)+\frac{x^{2}}{2 !} f^{\prime \prime \prime}(0)+\cdots+\frac{x^{n-1}}{(n-1) !} f^{(n)}(0)+\frac{x^{n}}{n !} f^{(n+1)}(0)+\cdots\right] .
\end{aligned}
$$


If $f^{\prime}(0)=F(0)$, then $f^{(p)}=F^{(p-1)}$ exist. So,

$$
\begin{aligned}
f\left(x+\varepsilon x^{*}\right)= & f(0)+\frac{x}{1 !} F(0)+\frac{x^{2}}{2 !} F^{\prime}(0)+\cdots+\frac{x^{n-1}}{(n-1) !} F^{(n-2)}(0)+\frac{x^{n}}{n !} F^{(n-1)}(0)+ \\
& \cdots+\varepsilon x^{*}\left[F(0)+\frac{x}{1 !} F^{\prime}(0)+\frac{x^{2}}{2 !} F^{\prime \prime}(0)+\cdots+\frac{x^{n-1}}{(n-1) !} F^{(n-1)}(0)+\frac{x^{n}}{n !} F^{(n)}(0)+\cdots\right] .
\end{aligned}
$$

Here, the first part is Taylor expansion of $f(x)$ at $x=0$ and the second part is Taylor expansion of $F(x)=f^{\prime}(x)$ at $x=0$. Thus,

$$
f\left(x+\varepsilon x^{*}\right)=f(x)+\varepsilon x^{*} f^{\prime}(x) .
$$

According to this, Taylor expansion $\cos \left(x+\varepsilon x^{*}\right), \sin \left(x+\varepsilon x^{*}\right)$ dual functions of them at $0=0+\varepsilon 0$ are [4]

$$
\begin{aligned}
\cos \left(x+\varepsilon x^{*}\right) & =\cos x-\varepsilon x^{*} \sin x, \\
\sin \left(x+\varepsilon x^{*}\right) & =\sin x+\varepsilon x^{*} \cos x .
\end{aligned}
$$

\section{Solution of Linear Differential Equations on Dual Space with Power Series}

In this study; second order homogeneous ordinary differential equations with variable and constant coefficients in dual space will be briefly referred to as linear differential equation. Solution of linear differential equations with power series is frequently used method [7,8]. The solutions in this method are in the from of Taylor series or Taylor expansion multiple by any power of $Z$. To have a solution, series has to be convergent. The series has only value at convergence interval. The addition of a convergent series is finite and defines a function. It may be found many works about these field of science in the literature $[2,5,11]$.

The general definition of a second order homogeneous linear differential equation in dual space is

$$
b_{2}(Z) Y^{\prime \prime}+b_{1}(Z) Y^{\prime}+b_{0}(Z) Y=0
$$

where $Y=Y(Z), Z=x+\varepsilon x^{*}$.

If each term of this equation is divided by $b_{2}(Z) \neq 0$, we find

$$
Y^{\prime \prime}+P(Z) Y^{\prime}+Q(Z) Y=0,
$$

where $P(Z)=\frac{b_{1}(Z)}{b_{2}(Z)}, Q(Z)=\frac{b_{0}(Z)}{b_{2}(Z)}$.

Definition 3.1. If both of constant functions $P(Z)$ and $Q(Z)$ of equation (3.1) is analytic in $Z_{0}$, then the point $Z_{0}$ is called ordinary point. If one of these points is not analytic in $Z_{0}$ is called singular point of (3.1) equation as $\left(Z-Z_{0}\right) P(Z)$ and $\left(Z-Z_{0}\right)^{2} Q(Z)$. If both coefficient functions of (3.1) equation $P(Z)$ and $Q(Z)$, is analytic in $Z_{0}, Z_{0}$ is called as ordinary point. If any of these functions is analytic in $Z_{0}$, in this case, $Z_{0}$ is a singular point of (3.1) equation. Specifically, if $Z_{0}$ is the regular singular point of equation (3.1) and both of $\left(Z-Z_{0}\right) P(Z)$ and $\left(Z-Z_{0}\right)^{2} Q(Z)$ is analytic in $Z_{0}, Z_{0}$ is regular singular point of (3.1) equation, otherwise, otherwise called non regular singular point.

Theorem 3.2. If $Z_{0}$ is ordinary point of second order linear differential equation (3.1), general solution of equation is

$$
Y=\sum_{n=0}^{\infty} A_{n}\left(Z-Z_{0}\right)^{n}=A_{0} Y_{1}(Z)+A_{1} Y_{2}(Z)
$$

in the interval which contains $Z_{0}$ points and where $A_{0}$ and $A_{1}$ are arbitrary dual fixed numbers, $Y_{1}(Z)$ and $Y_{2}(Z)$ are analytic functions in $Z_{0}$. If the ordinary point is $Z_{0} \neq 0$, serial solutions of the new equation are obtained by using $T=Z-Z_{0}$ changing variables method. As a result, the serial solution of the equation is obtained by using changing the variables again $T=Z-Z_{0}$. If both $P(Z)$ and $Q(Z)$ in (3.1) are constants, each point is ordinary point and the power series method can be applied to each point.

Example 3.3. The solution of

$$
\frac{d^{2} Y}{d Z^{2}}+Y=0
$$

constant coefficients linear differential equation with power series where $Y=Y(Z), Z=x+\varepsilon x^{*}$ at dual point $Z_{0}$ neighbourhood. Dual point $Z_{0}$ is a ordinary point of this equation. So, the solution of this equation is

$$
Y=\sum_{n=0}^{\infty} A_{n} Z^{n}=A_{0}\left(x^{n}+\varepsilon n x^{*} x^{n-1}\right)
$$


If

$$
\begin{aligned}
\frac{d Y}{d Z} & =\sum_{n=0}^{\infty} n A_{n} Z^{n-1}=\sum_{n=0}^{\infty} n A_{n}\left(x^{n-1}+\varepsilon(n-1) x^{*} x^{n-2}\right) \\
\frac{d^{2} Y}{d^{2} Z} & =\sum_{n=0}^{\infty} n(n-1) A_{n} Z^{n-2}=\sum_{n=0}^{\infty} n(n-1) A_{n}\left(x^{n-2}+\varepsilon(n-2) x^{*} x^{n-3}\right)
\end{aligned}
$$

are written in (3.2) and making some arrangement, we find

or

$$
\sum_{n=0}^{\infty}\left[(n+2)(n+1) A_{n+2}+A_{n}\right] x^{n}=\sum_{n=0}^{\infty}\left[(n+2)(n+1) A_{n+2}+A_{n}\right] \varepsilon n x^{*} x^{n-1}=0
$$

Thus, the solution from

$$
\sum_{n=0}^{\infty}\left[(n+2)(n+1) A_{n+2}+A_{n}\right] Z^{n}=0
$$

If each ower of constant in $Z$ is equal to zero, the reduction relation is

$$
\begin{gathered}
A_{n+2}=-\frac{1}{(n+2)(n+1)} A_{n}, n=0,1,2, \ldots, \\
A_{n}=\left\{\begin{array}{cc}
\frac{(-1)^{k}}{(2 k) !} A_{0}, & n=k \\
\frac{(-1)^{k}}{(2 k+1) !} A_{1}, n=2 k+1
\end{array} .\right.
\end{gathered}
$$

$$
\begin{aligned}
Y(Z) & =A_{0} \sum_{k=0}^{\infty} \frac{(-1)^{k}}{(2 k) !} Z^{2 k}+A_{1} \sum_{k=0}^{\infty} \frac{(-1)^{k}}{(2 k+1) !} Z^{2 k+1} \\
& =A_{0} \cos Z+A_{1} \sin Z \\
& =A_{0} \cos \left(x+\varepsilon x^{*}\right)+A_{1} \sin \left(x+\epsilon x^{*}\right)
\end{aligned}
$$

is

$$
Y=A_{0} \cos x+A_{1} \sin x+\varepsilon x^{*}\left(-A_{0} \sin x+A_{1} \cos x\right) .
$$

Example 3.4. The solution of variable coefficients linear differential equation with power series where

$$
\begin{aligned}
& Y=Y(Z), Z=x+\varepsilon x^{*}, \\
& \frac{d^{2} Y}{d Z^{2}}-Z \frac{d Y}{d Z}+2 Y=0
\end{aligned}
$$

at dual point $Z_{0}=0$ neighbourhood. Dual point $Z_{0}=0$ is an ordinary point of this equation. So, there is a

$$
Y=\sum_{n=0}^{\infty} A_{n} Z^{n}=\sum_{n=0}^{\infty} A_{n}\left(x^{n}+\varepsilon n x^{*} x^{n-1}\right)
$$

solution for this equation. If the equations

$$
\begin{gathered}
\frac{d Y}{d Z}=\sum_{n=0}^{\infty} n A_{n} Z^{n-1}=\sum_{n=0}^{\infty} n A_{n}\left(x^{n-1}+\varepsilon(n-1) x^{*} x^{n-2}\right), \\
\frac{d^{2} Y}{d Z^{2}}=\sum_{n=0}^{\infty} n(n-1) A_{n} Z^{n-2}=\sum_{n=0}^{\infty} n(n-1) A_{n}\left(x^{n-2}+\varepsilon(n-2) x^{*} x^{n-3}\right)
\end{gathered}
$$

are written in (3.3) and with some arrangement, we find

or

$$
\sum_{n=0}^{\infty}\left[(n+2)(n+1) A_{n+2}-(n-2) A_{n}\right] x^{n}+\sum_{n=0}^{\infty}\left[(n+2)(n+1) A_{n+2}-(n-2) A_{n}\right] \varepsilon n x^{n-1} x^{*}=0
$$

$$
\sum_{n=0}^{\infty}\left[(n+2)(n+1) A_{n+2}-(n-2) A_{n}\right] Z^{n}=0
$$


If each power of constant of $Z$ is equal to zero, the reduction relation is

$$
A_{n+2}=\frac{n-2}{(n+2)(n+1)} A_{n}, n=0,1,2, \ldots .
$$

From this relation we get $A_{4}=0$. It is clear that all of even indis coffients are zero. That is on the otherside,

Thus the solution

$$
A_{3}=-\frac{1}{6} A_{1}, A_{5}=-\frac{1}{120} A_{1}, A_{7}=-\frac{1}{1680} A_{1}, \ldots .
$$

$$
\begin{aligned}
Y(Z)= & A_{0}\left(1-Z^{2}\right)+A_{1}\left(Z-\frac{1}{6} Z^{3}-\frac{1}{120} Z^{5}-\frac{1}{1680} Z^{7}-\cdots\right) \\
= & A_{0}\left[1-\left(x+\varepsilon x^{*}\right)^{2}\right]+A_{1}\left[\left(x+\varepsilon x^{*}\right)-\frac{1}{6}\left(x+\varepsilon x^{*}\right)^{3}-\frac{1}{120}\left(x+\varepsilon x^{*}\right)^{5}-\frac{1}{1680}\left(x+\varepsilon x^{*}\right)^{7}-\cdots\right] \\
= & A_{0}\left(1-x^{2}\right)+A_{1}\left(x-\frac{1}{6} x^{3}-\frac{1}{120} x^{5}-\frac{1}{1680} x^{7}-\cdots\right) \\
& +\varepsilon x^{*}\left[-2 A_{0} x+A_{1}\left(1-\frac{1}{2} x^{2}-\frac{1}{24} x^{4}-\frac{1}{240} x^{6}-\cdots\right)\right] .
\end{aligned}
$$

Example 3.5 (Regular singular points and method of Frobenius). If dual point $Z_{0}=x_{0}+\varepsilon x_{0}^{*}$ is not a ordinary point of second order homogenous linear differential equation

$$
Y^{\prime \prime}+P(Z) Y^{\prime}+Q(Z) Y=0
$$

but both $\left(Z-Z_{0}\right) P(Z)$ and $\left(Z-Z_{0}\right)^{2} Q(Z)$ are analytic in $Z_{0}$, then $Z_{0}$ is a regular singular point of (3.4) equation.

General Solution:

Method of Frobenius always give a solution

$$
Y_{1}(Z)=Z^{\lambda_{1}} \sum_{n=0}^{\infty} A_{n}\left(\lambda_{1}\right) Z^{n}
$$

of (3.4) equation. General solution is in the form $Y=c_{1} Y_{1}(Z)+c_{2} Y_{2}(Z)$ where $c_{1}$ and $c_{2}$ are arbitrary constants, $Y^{\prime \prime}(Z)$ is a solution in (3.4) which is linear independent from $Y_{1}(Z)$. The method of finding this second solution is depended on the relation in between two roots of index equation.

i) If $\lambda_{1}-\lambda_{2}$ is not an integer, it is $Y_{2}(Z)=Z^{\lambda_{2}} \sum_{n=0}^{\infty} A_{n}\left(\lambda_{2}\right) Z^{n}$.

ii) If $\lambda_{1}=\lambda_{2}$, then it is

$$
Y_{2}(Z)=Y_{1}(Z) \operatorname{In} Z+Z^{\lambda_{1}} \sum_{n=0}^{\infty} B_{n}\left(\lambda_{1}\right) Z^{n} .
$$

To find this soluion, making recurrence formul according $\lambda$ to where constant $A_{0}$ is arbitrary, this formul is used for finding $A_{n}(n \geq 1)$ constants the type of $\lambda$ and $A_{0}$. This $A_{n}$ values are written in the equation to find a $Y(\lambda, Z)$ function which is depended to $\lambda$ and $Z$ variables. Thus we find, $Y_{2}(Z)=\left.\frac{d Y(\lambda, Z)}{d \lambda}\right|_{\lambda=\lambda_{1}}$

iii) If $\lambda_{1}-\lambda_{2}=N$ (a positive integer), then

$$
Y_{2}(Z)=d_{-1} Y_{1}(Z) I n Z+Z^{\lambda_{2}} \sum_{n=0}^{\infty} d_{n}\left(\lambda_{2}\right) Z^{n} .
$$

To find this solution, Frobenius method is tried with $\lambda_{2}$ firstly. If this gives a second solution, then this solution is $Y_{2}(Z)$ which is in the form of (3.5) where $d_{-1}=0$. Otherwise, to find, $Y(\lambda, Z)$, second case is used. Then, we get

$$
Y_{2}(Z)=\left.\frac{d}{d \lambda}\left[\left(\lambda-\lambda_{2}\right) Y(\lambda, Z)\right]\right|_{\lambda=\lambda_{2}} \text {. }
$$

Example 3.6 (The solution of hypergeometric differential equation's at regular singular point $Z_{0}=0$ neighbourhood). At the neighbourhood of singular point, the solution of

$$
Z(1-Z) Y^{\prime \prime}+[\gamma-(\alpha+\beta+1) Z] Y^{\prime}-\alpha \beta Y=0,
$$

where $\alpha, \beta$ and $\gamma$ are real constants, hypergeometric differential equation at the neighbourhood of $Z_{0}=0$ regular singular point with using Frobenius method: 
There exists a solution $Y=\sum_{n=0}^{\infty} A_{n} Z^{m+n}$ of equation (3.6) at the neighbourhood of point $Z_{0}=0$ where $A_{0} \neq 0$. With derive and making some arrangements, we find

$$
\begin{aligned}
{\left[m^{2}+(\gamma-1) m\right] A_{0} Z^{m-1}+\sum_{n=0}^{\infty}\left\{(m+n+1)(m+n+\gamma) A_{n+1}\right.} \\
\left.-[(m+n)(m+n+\alpha+\beta)+\alpha \beta] A_{n}\right\} Z^{m+n}=0 .
\end{aligned}
$$

Here,

$$
m^{2}+(\gamma-1) m=0
$$

and index equation

$$
\begin{gathered}
A_{n+1}=\frac{(m+n)(\alpha+\beta+m+n)+\alpha \beta}{(m+n+1)(m+n+\gamma)} A_{n}, n=0,1,2, \ldots, \\
A_{n+1}=\frac{(\alpha+m+n)(\beta+m+n)}{(m+n+1)(m+n+\gamma)} A_{n}, n=0,1,2, \ldots
\end{gathered}
$$

is to obtain where $A_{0} \neq 0$. The roots of index equation (3.7) are $m_{1}=0$ and $m_{2}=1-\gamma$.

i) Reduction relation for $m_{1}=0$ from (3.8) is

$$
A_{n+1}=\frac{(\alpha+n)(\beta+n)}{(1+n)(\gamma+n)} A_{n}, n=0,1,2, \ldots .
$$

If we give the values of $0,1,2, \ldots, n-1$ to $n$ at (3.9) and multiple side by side, we find the cofficients

$$
A_{n}=\frac{\alpha(\alpha+1) \ldots(\alpha+n-1) \beta(\beta+1) \ldots(\beta+n-1)}{n ! \gamma(\gamma+1) \ldots(\gamma+n-1)} A_{0}
$$

or

$$
A_{n}=\frac{(\alpha)_{n}(\beta)_{n}}{n !(\gamma)_{n}}
$$

To make the denominatör nonzero, we should take $\gamma \neq 0,-1,-2, \ldots$ So if we take $a_{0}=1$, a solution of equation (3.6) is

$$
\begin{aligned}
Y_{1}(Z) & =F(\alpha, \beta ; \gamma ; Z)=\sum_{n=0}^{\infty} \frac{(\alpha)_{n}(\beta)_{n}}{(\gamma)_{n}} \frac{Z^{n}}{n !} \\
& =\sum_{n=0}^{\infty} \frac{(\alpha)_{n}(\beta)_{n}}{(\gamma)_{n}} \frac{\left(x+\varepsilon x^{*}\right)^{n}}{n !} \\
& =\sum_{n=0}^{\infty} \frac{(\alpha)_{n}(\beta)_{n}}{(\gamma)_{n}} \frac{\left(x+\varepsilon n x^{*} x^{n-1}\right)}{n !} \\
& =F(\alpha, \beta ; \gamma ; x)+\varepsilon x^{*} \frac{\alpha \beta}{\gamma} \sum_{n=0}^{\infty} \frac{(\alpha+1)_{n}(\beta+1)_{n}}{(\gamma+1)_{n}} \frac{x^{n}}{n !} \\
& =F(\alpha, \beta ; \gamma ; x)+\varepsilon x^{*} \frac{d}{d x} F(\alpha, \beta ; \gamma ; x) .
\end{aligned}
$$

ii) Now let's find the second solution. Reduction relation for $m_{2}=1-\gamma$ from (3.8) is

$$
A_{n+1}=\frac{(\alpha+1-\gamma+n)(\beta+1-\gamma+n)}{(n+2-\gamma)(n+1)} A_{n}, n=0,1,2, \ldots .
$$

At this relation, if we give the values of $0,1,2, \ldots, n-1$ to $n$ and multiple side bye side, we find

$$
A_{n}=\frac{(\alpha-\gamma+1)(\alpha-\gamma+2) \cdots(\alpha-\gamma+n)(\beta-\gamma+1)(\beta-\gamma+2) \cdots(\beta-\gamma+n)}{n !(2-\gamma)(3-\gamma) \cdots(n+1-\gamma)} A_{0}
$$

or

$$
A_{n}=\frac{(\alpha-\gamma+1)_{n}(\beta-\gamma+1)_{n}}{n !(2-\gamma)_{n}} A_{0}, n=1,2, \ldots
$$


To make the denominator nonzero, we should take $\gamma \neq 2,3,4, \ldots$. Accordingly, if we take $a_{0}=1$, the second solution of equation (3.6) is

$$
\begin{aligned}
Y_{2}(Z)= & Z^{1-\gamma} \sum_{n=0}^{\infty} \frac{(\alpha+1-\gamma)_{n}(\beta+1-\gamma)_{n}}{(2-\gamma)_{n}} \frac{Z^{n}}{n !} \\
= & \left(x+\varepsilon x^{*}\right)^{1-\gamma} \sum_{n=0}^{\infty} \frac{(\alpha+1-\gamma)_{n}(\beta+1-\gamma)_{n}}{(2-\gamma)_{n}} \frac{\left(x+\varepsilon x^{*}\right)^{n}}{n !} \\
= & x^{1-\gamma} F(\alpha-\gamma+1, \beta-\gamma+1 ; 2-\gamma ; x)+\varepsilon x^{*}\left[(1-\gamma) x^{-\gamma} F(\alpha-\gamma+1, \beta-\gamma+1 ; 2-\gamma ; x)\right. \\
& \left.+x^{1-\gamma} \frac{d}{d x} F(\alpha-\gamma+1, \beta-\gamma+1 ; 2-\gamma ; x)\right] .
\end{aligned}
$$

If $\gamma=1$, there exists $Y_{1}$ and $Y_{2}$, but they are not linear independet.

If $\gamma=2,3,4, \ldots$ there exists $Y_{1}$, but not $Y_{2}$.

If $\gamma \neq 0,-1,-2, \ldots$ there exists $Y_{2}$, but not $Y_{1}$.

So, if $\gamma$ is not an integer, then the solutions $Y_{1}$ and $Y_{2}$ of (3.10) and (3.11) respectively are the linear independent solutions of hypergeometric differential equation. Thus, general solution (3.6) is,

$$
\begin{aligned}
Y= & A Y_{1}+B Y_{2} \\
= & {\left[A F(\alpha, \beta ; \gamma ; x)+B x^{1-\gamma} F(\alpha-\gamma+1, \beta-\gamma+1 ; 2-\gamma ; x)\right]+\varepsilon x^{*}\left\{A \frac{d}{d x} F(\alpha, \beta ; \gamma ; x)\right.} \\
& \left.+B\left[(1-\gamma) x^{-\gamma} F(\alpha-\gamma+1, \beta-\gamma+1 ; 2-\gamma ; x)+x^{1-\gamma} \frac{d}{d x} F(\alpha-\gamma+1, \beta-\gamma+1 ; 2-\gamma ; x)\right]\right\},
\end{aligned}
$$

where $A$ and $B$ are arbitrary constants.

If $\alpha$ or $\beta$ are zero or minus integer constants, then, $Y_{1}$ is a polinomial which are $\gamma=1,2,3, \ldots$ If $\alpha-\gamma+1$ or $\beta-\gamma+1$ are zero or minus integer constants, then, $Y_{2}$ can be converted polinomial which are $\gamma=1,0,-1,-2, \ldots$ and this polinomial multiplied by $x^{1-\gamma}$. Thus, in these cases, all the terms after certain terms of the series (3.10) and (3.11) are zero. These polynomials are called as hypergeometric polynomials. Except of these cases, if $\gamma$ is an integer, one of the solutions is logarithmic.

In the same manner, at the regular singular point $x=1$, two solutions of (3.6) are given as follows:

$$
\begin{aligned}
& Y_{1}=F(\alpha, \beta ; \alpha+\beta-\gamma+1 ; 1-x), \\
& Y_{2}=(1-x)^{\gamma-\alpha-\beta} F(\gamma-\alpha, \gamma-\beta ; \gamma-\alpha-\beta+1 ; 1-x) .
\end{aligned}
$$

\section{Conclusion}

In this study, solutions of second order linear homogeneous ordinary differential equations with variable coefficients and second order linear homogeneous ordinary differential equations with constant coefficients by using dual numbers, which are the elements of dual space have been discussed. In dual space, the solutions of second order linear homogeneous ordinary differential equations with variable and constant coefficients have been given by power series and their simulations have been exemplified. It has been observed that the results are similar to the solutions of the second order linear homogeneous variable and constant coefficient ordinary differential equations in real space.

\section{CONFLICTS OF INTEREST}

The authors declare that there are no conflicts of interest regarding the publication of this article.

\section{REFERENCES}

[1] Asil, V., Aydın, M.E., Küçükarslan, Z., Çınar, G., Tatlıpınar, S., The solution of differential equations using dual functions, e-Journal of NWSA, 4(4)(2009), Article number: 3A0012. 1

[2] Aydın, M., Kuryel, B., Gündüz, G., Oturanç, G., Differential Equations and Applications, E.Ü. Textbooks Publications of Faculty of Engineering, No.14, İzmir, 2001.3 
[3] Hacısalihoğlu, H.H., General dual motion of n moving reference frames, Communications de la Faculte des Sciences De l'Universite d'Ankara, (1971), 71-85. 1

[4] Hacısalihoğlu, H.H., Motion Geometry and Quaternions Theory, Gazi University Faculty of Arts and Science Publications, Math. 2, 1983. 1, $2.2,2.3,2.5,2.6,2.7,2.8,2.9,2$

[5] Jiménez, N.G, Torba, S.M., Spectral parameter power series representation for solutions of linear system of two first order differential equations, Applied Mathematics and Computation, 370(124911), (2020), 1-10. 3

[6] Mak, M., Koru Yücekaya, G., Çetinkaya, A., On the solutions of differential equations with series of powers in dual space, VII. National Geometry Symposium, 07-10 July 2009, Kırşehir, Turkey, (2009). 1

[7] Ritger, Paul D., Rose, Nicholas J., Differential Equations with Applications, NY, Dover Publications, IX., 2000. 3

[8] Ross, Shepley L., Differential Equations, John Wiley and Sons, Inc., 1974. 3

[9] Veldkamp, G.R., On the use dual numbers, vectors and matrices in instantaneous spatial kinematics, Mech. Mach Theory, 11(1976), $141-156$. $2.1,2.4$

[10] Yang, A.T., Application of Quaternion Algebra and Dual Numbers to Analysis of Spatial Mechanisms, Doctoral Dissertation, Columbia University, 1963. 2.1, 2.4

[11] Yaşar, İ.B., Differential Equations and Applications, Siyasal Bookstore, 2nd. Ed., Ankara, 1999. 3 